\title{
Up-scaling RP measures for emergency and post-emergency and the need for refinements further to the ICRP general recommendations
}

\author{
S. SAINT-PIERRE
}

ABSTRACT Over the last few decades, the steady progress achieved in reducing planned exposures of both workers and the public has been admirable in the nuclear sector. However, the disproportionate focus on tiny public exposures and radioactive discharges associated with normal operations came at a high price, and the quasi-denial of a risk of major accident and related weaknesses in emergency preparedness and response came at even higher price. Fukushima has unfortunately taught us that radiological protection (RP) for emergency and post-emergency can be much more than a simple evacuation that lasts 24 to 48 hours with people safely returning to their homes shortly afterward. On optimization of emergency and post-emergency exposures, the only "show in town" in terms of international RP policies improvements has been the issuance of the ICRP's new general recommendations. However, no matter how genuine these improvements were, they have not been "road tested" to the practical reality of severe accidents. Post-Fukushima, there is a compelling case to review the practical adequacy of key RP notions such as optimization, evacuation, sheltering, reference levels for workers and the public, and to amend these notions with a view to make the international RP system more useful in the event of a severe accident. In pursuing further improvements to the international RP system, it should be clearly borne in mind that the system is generally based on protection against the risk of cancer and hereditary diseases. The system also protects against deterministic non-cancer effects on tissues and organs. In seeking refinements of such protection notions, we invite ICRP to pay increased attention to the fact that a continued balance must be struck between beneficial activities which cause exposures, and protection. The global nuclear industry is committed to help overcoming the above key RP issues as part of the RP community's upcoming international deliberations towards a more efficient international RP system.

Keywords: International radiological protection system / optimization / planned exposure / emergency and post-emergency exposures / tissue reactions and non-cancer effect

WNA - World Nuclear Association, Carlton House, 22a. St. James's Square, London, United Kingdom. 


\section{Introduction}

Over the last few decades, the steady progress achieved in reducing planned exposures of both workers and the public have been admirable in the nuclear sector. However, the disproportionate focus on tiny public exposures and radioactive discharges associated with normal operations of nuclear industrial facilities came at a high price, and the quasi-denial of a risk of major accident and related weaknesses in emergency preparedness and response came at even higher price. Fukushima has unfortunately taught us that radiological protection (RP) for emergency and post-emergency can be much more than a simple evacuation that lasts 24 to 48 hours with people safely returning to their homes shortly afterward. This paper addresses global nuclear industry views on RP for emergency and post-emergency. It also provides views on the emerging topic of tissue reactions and non-cancer effects as part of the international RP system.

\section{Radiological protection of emergency and post-emergency}

Post-Fukushima, nuclear regulators and nuclear operators are currently working hard at reviewing and up-scaling their emergency preparedness and response for possible severe nuclear accidents at nuclear power sites. Amongst others, important issues being re-examined include: source term analysis, evacuation, sheltering, and monitoring and availability of protective equipment, etc. In this regard, the national agendas are the top priority and individual nations are moving faster than progress at the international level meaning that changes will be made at nuclear power plants in order to address changes in national requirements way before any change in international policies and standards. Regarding post-emergency activities, a number of issues are expected to arise relative to clean-up criteria for contamination, conditions for allowing people to return in their homes after evacuation, etc.

Regarding international RP policies, standards, and programmes for emergency and post-emergency, pre-Fukushima, they were rather scaled for less severe events of shorter duration. The new ICRP recommendations issued in late 2007 (ICRP, 2007) have brought forward significant changes in this regard. On the positive side, these recommendations were "the only show in town" in terms of laying out new RP policies that account for possible severe accidents of considerable duration (e.g. much more than just 24 or 48 hours). However, no matter how genuine these improvements were, the new ICRP recommendations measures on $\mathrm{RP}$ for emergency and post-emergency are rather tutorial in style and their application has not been "road tested" against the practical reality of a severe accident such as the Fukushima one. Moreover, the new ICRP recommendations have been incorporated within the recent revision of the IAEA Basic Safety Standards (BSS) for Protection against Ionizing Radiation and for the Safety of Radiation Sources (IAEA, 2011) completed in March 2011 after a few years of review. One of the industry regrets is not to have signaled this key issue sufficiently early as part of discussions concerning the IAEA's BSS revision. 
Post-Fukushima and subsequent to the above-mentioned priority given to national agendas, there will be a compelling case to review and revise current RP policies, standards and programmes for emergency and post-emergency in light of the lessons to be learned from the comprehensive analyses of the Fukushima accident. As part of this, the practical adequacy of key RP notions such as optimization, "voluntary" responders, emergency dose limits, transition between an emergency exposure situation and an existing exposure situation, implications for evacuation, sheltering and post-emergency, and reference levels for workers and the public, will have to be carefully re-examined. The list below illustrates the types of issues that may need relevant for such a review:

- To which extent is optimization practically applicable during an accident and afterward?

- Is the notion of "voluntary" responders adequate? If the most experienced staff from a nuclear site is not willing to respond voluntary, who else should, or could, ensure this vital function?

- International guidance on emergency limits includes key subtleties that should not be overlooked. For life saving, ICRP recommends no dose restrictions if benefit to others outweighs rescuer's risk. For other urgent rescue operations, the limit is set at $500 \mathrm{mSv}$ whereas it is set at $100 \mathrm{mSv}$ for other rescue operations. If any, the benefit of reducing emergency limit values is unclear. Would an activity involving five experienced responders instead of a single responder, represent an RP improvement?

- With an emergency phase that spans over several months, how should the transition - including all applicable rules - between the emergency phase and post-emergency (existing) phase be managed?

- What do the dose reference levels of 1.20 and $100 \mathrm{mSv} / \mathrm{y}$ really mean in terms of public health and of societal issues in relation to allowing or disallowing evacuees to return to their homes? After a major accident, as you cannot know a priori what you would end-up with, is international guidance which suggests a long term goal of $1 \mathrm{mSv} / \mathrm{y}$ helpful?

The Fukushima accident has already triggered a first IAEA review of all IAEA Safety Standards. This review has been launched by the IAEA's Director General in September 2011 with a report due one year later. It puts the focus on reviewing the IAEA Safety Requirements - as opposed to Safety Guides. A related IAEA action plan puts emphasis on the safety of nuclear reactor designs as well as on the safety of storage of used nuclear fuel. The review also encompasses emergency preparedness and response measures, which in turn embed related international RP policies and standards for emergency and post-emergency.

Moreover, once reliable lessons will be learned from in-depth analyses of the Fukushima accident, it is expected that this may trigger a further review of international RP policies and standards for emergency and post-emergency. In brief, we can expect that industry and governments are in for a long journey in relation to changes in RP policies and standards. 
Over the coming years, it is therefore important for industry to be continuously involved through a constructive dialogue with leading international RP agencies, governmental agencies and others on these issues. Differences between countries/companies will be part of the picture which will need to be carefully accounted for at the international level.

\section{Tissue reactions and non-cancer effects}

Because the key topic of tissue reactions and non-cancer effects has potential to be a "game changer" in the international RP system of the future, industry and governments must pay increased attention to its evolution. In brief, the ICRP and its system of protection were created after the discovery of health casualties from the increased use of X-rays. The system is generally based on protection against the risk of cancer and hereditary diseases. In addition, it also covers protection against deterministic effects on tissues and organs that differ from cancer (noncancer effects).

By analogy, the rapid increase and wider use of radiation and nuclear technology in the medical sector may bring a "quantum" change in the international RP system within this decade. The main reason for such a change is that this significantly enhanced use of radiation and nuclear technology in the medical sector - for beneficial reasons (e.g. diagnostic and therapy) - will likely lead to the emergence of statistically significant radiation-induced effects (stochastic effects) other than cancer such as cataracts, blood circulatory diseases, and cardiovascular diseases, amongst others. In other words, the notion of thresholds for deterministic effects on organs and tissues will likely change as refined scientific knowledge on radiation effects and protection is gained. This is one reason why ICRP has recently evolved from the notion of "deterministic effects" to the notion of "tissue reactions and non-cancer effects".

Judgment calls on tissue reactions and non-cancer effects will need to be done very cautiously as it can raise significant imbalance in the overall RP system. Evolving from a system of general protection against cancer and mortality to a system that encompasses a refined level of protection against the incidence of more or less severe non-fatal health diseases (with many that are curable) needs to involve well considered judgement calls. For example, if a general level of protection of $5 \%$ per Sievert $(5 \% / \mathrm{Sv})$ is provided against general cancer, protection against the incidence of a given more or less severe non-cancer effects (cataracts or blood circulatory diseases for example) need to be carefully chosen in a coherent manner.

As large workforces are part of the nuclear industry, it goes without saying that the evolution of stochastic health risks related to non-cancer effects is a source of concern for the nuclear industry. Similarly, such a concern would be amplified if a major nuclear accident results in the exposure of large populations. 
Above all, in seeking refinements in protection against tissue reactions and non-cancer effects, we invite ICRP to pay increased attention to the fact that a continued balance must be struck between beneficial activities which cause exposures, and protection. The global nuclear industry is committed to help overcoming the above key RP issues as part of the RP community's upcoming deliberations towards a more efficient international RP system.

\section{Conclusions}

On RP for emergency and post-emergency exposures, RP upgrades are getting deployed at nuclear power plants in relation to emergency preparedness and response for possible severe nuclear accidents. This is a top priority for both industry and national regulators. Post-Fukushima, international RP policies and standards will need to be further reviewed and improved as a subsequent priority. For sound improvements that can be practically implemented on such important topics, the industry input will be key and industry is prepared to fulfil this key role gradually over time as robust lessons learned will emerge from comprehensive analyses of the Fukushima accident.

In pursuing further improvements to the international RP system, it should be clearly borne in mind that the system is generally based on protection against the risk of cancer and hereditary diseases. In addition, the system also protects against deterministic non-cancer effects on organs and tissues. In seeking refinements of such protection notions, we invite ICRP to pay increased attention to the fact that a continued balance must be struck between beneficial activities which cause exposures, and protection.

The global nuclear industry is committed to help overcoming the above key $\mathrm{RP}$ issues as part of the RP community's upcoming international deliberations towards a more efficient international RP system.

\section{REFERENCES}

IAEA (2011) Basic Safety Standards (BSS) for Protection against Ionizing Radiation and for the Safety of Radiation Sources, Draft 5.0, 21 March 2011, Vienna, Austria.

ICRP Publication 103 (2007) The 2007 Recommendations of the International Commission of Radiological Protection, Ann. ICRP 37 (2-4). 